\title{
AN EXTENSION OF THE HERMITE-HADAMARD INEQUALITY THROUGH SUBHARMONIC FUNCTIONS*
}

\author{
MIHAI MIHĂILESCU and CONSTANTIN P. NICULESCU \\ Department of Mathematics, University of Craiova, 200585 Craiova, Romania \\ e-mail:mmihailes@yahoo.com cniculescu47@yahoo.com
}

(Received 15 December, 2006; accepted 31 March, 2007)

\begin{abstract}
In this paper we obtain a Hermite-Hadamard type inequality for a class of subharmonic functions. Our proofs rely essentially on the properties of elliptic partial differential equations of second order. Our study extends some recent results from [1], [2] and [6].
\end{abstract}

2000 Mathematics Subject Classification. 35R45, 35J15, 35J60.

1. Introduction and main result. The classical Hermite-Hadamard inequality provides a valuable two-sided estimate of the mean value of a continuous convex function $f:[a, b] \rightarrow \mathbb{R}:$

$$
f\left(\frac{a+b}{2}\right) \leq \frac{1}{b-a} \int_{a}^{b} f(t) d t \leq \frac{f(a)+f(b)}{2} .
$$

This fact was extended within the Choquet theory to the general framework of continuous convex functions on a compact convex subset $K$ (of a metrizable locally convex space) and of Borel probability measures $\mu$ on $K$. See [7] for details. Is it possible to extend Choquet's theory to the more general case of signed measures? Recently, A. Florea and C. P. Niculescu [2] solved completely the case of compact intervals, based on earlier work due to A. M. Fink [1]. More precisely, they provided a full characterization of those signed Borel measures $\mu$ on $[a, b]$ such that $\mu([a, b])>0$ and

$$
\begin{aligned}
f\left(x_{\mu}\right) & \leq \frac{1}{\mu([a, b])} \int_{[a, b]} f(t) d \mu(t) \\
& \leq \frac{b-x_{\mu}}{b-a} \cdot f(a)+\frac{x_{\mu}-a}{b-a} \cdot f(b),
\end{aligned}
$$

for all continuous convex functions $f:[a, b] \rightarrow \mathbb{R}$, where

$$
x_{\mu}=\frac{1}{\mu([a, b])} \int_{[a, b]} t d \mu(t)
$$

is the barycenter of $\mu$. Besides the case of Borel probability measures, other examples are offered by the family $d \mu=\left(x^{2}+\lambda\right) d x$ on $[-1,1]$, when $\lambda \geq-1 / 6$. See [2].

\footnotetext{
*Correspondence address: Mihai Mihăilescu, Department of Mathematics, University of Craiova, 200585 Craiova, Romania. E-mail: mmihailes@yahoo.com
} 
A natural method to extend results regarding convex functions of one real variable to several variable functions is due to P. Montel [5] and appeals to subharmonic functions. By a subharmonic function $u$ defined on a domain $D \subset \mathbb{R}^{N}(N \geq 2)$, we understand a $C^{2}$-differentiable function on $D$ with the property that

$$
\Delta u \geq 0, \quad \text { in } D,
$$

where $\Delta=\sum_{i=1}^{N} \frac{\partial^{2}}{\partial x_{i}^{2}}$ denotes the Laplace operator.

C. P. Niculescu and L.-E. Persson gave in [6] an extension of the HermiteHadamard inequality to this context. They proved that if $\Omega \subset \mathbb{R}^{N}$ is a bounded open subset with smooth boundary, $u \in C^{2}(\Omega) \cap C^{1}(\bar{\Omega})$ is subharmonic and $\varphi \in$ $C^{2}(\Omega) \cap C^{1}(\bar{\Omega})$ is a solution of the problem

$$
\begin{cases}\Delta \varphi=1, & \text { for } x \in \Omega \\ \varphi=0, & \text { for } x \in \partial \Omega\end{cases}
$$

then

$$
\int_{\Omega} u d V<\int_{\partial \Omega} u(\nabla \varphi \cdot n) d S
$$

except for harmonic functions (when equality occurs).

In the particular case when $\Omega$ is the open ball $B_{R}(a)$ (centered in $a$ and of radius $R$ ) in $\mathbb{R}^{3}$, the maximum principle for elliptic problems combined with the above result yield the following Hermite-Hadamard type inequality for subharmonic functions (which are not harmonic):

$$
u(a) \leq \frac{1}{\operatorname{Vol} \bar{B}_{R}(a)} \iiint_{\bar{B}_{R}(a)} u(x) d V<\frac{1}{\operatorname{Area} S_{R}(a)} \iint_{S_{R}(a)} u(x) d S .
$$

Formula (3) shows that for the measure $d \mu=\frac{1}{\operatorname{Vol}_{\bar{B}_{R}(a)}} d V$ there exists a measure $d \nu=\frac{1}{\text { Area } S_{R}(a)} d S$ concentrated on the boundary of $\Omega=\bar{B}_{R}(a)$ such that

$$
\int_{\Omega} f d \mu \leq \int_{\partial \Omega} f d v
$$

for all subharmonic functions $f$.

In this paper we prove that a similar result works when the Laplace operator is replaced by a strictly elliptic self-adjoint linear differential operator of second order which admits a Green function.

More precisely, we shall deal with operators $L: C^{2}(\Omega) \rightarrow C(\Omega)$ defined by

$$
L u=\sum_{i=1}^{N} \sum_{j=1}^{N} a_{i j}(x) \frac{\partial^{2} u}{\partial x_{i} \partial x_{j}}+\sum_{i=1}^{N} b_{i}(x) \frac{\partial u}{\partial x_{i}}+c(x) u,
$$

where $a_{i j}(x)=a_{j i}(x) \in C^{1}(\Omega), b_{i}(x) \in C(\Omega)$ and $c(x) \in C(\Omega)$ is a negative function in $\Omega$.

As above, $\Omega \subset \mathbb{R}^{N}(N \geq 2)$ will be a bounded domain with smooth boundary. 
We assume that $L$ is strictly elliptic on $\Omega$, i.e.

$$
\sum_{i=1}^{N} \sum_{j=1}^{N} a_{i j}(x) \xi_{i} \xi_{j} \geq \lambda|\xi|^{2}, \quad \forall x \in \Omega, \forall\left(\xi_{1}, \ldots, \xi_{N}\right) \in \mathbb{R}^{N},
$$

for some positive constant $\lambda$ and self-adjoint, i.e.

$$
b_{i}(x)=\sum_{j=1}^{N} \frac{\partial a_{i j}(x)}{\partial x_{j}}, \quad \forall i=1, \ldots, N, \quad \forall x \in \Omega .
$$

For the strictly elliptic, self-adjoint, linear second order differential operator $L$ on the domain $\Omega$ we introduce the Green function $G: \bar{\Omega} \times \bar{\Omega} \rightarrow \mathbb{R}$ as a function having the following three properties:

(G1) $G(x, \xi)|x-\xi|^{N-2}$ is a bounded function of $\xi$ and has a positive lower bound for $\xi$ near $x$;

(G2) $L_{\xi}[G(x, \xi)]=0$ in $\Omega$ for $\xi \neq x$. The notation $L_{\xi}$ means that we apply the operator $L$ to the coordinates $\left(\xi_{1}, \ldots, \xi_{N}\right)$ of $\xi$ in $G(x, \xi)$ and keep $x=\left(x_{1}, \ldots, x_{N}\right)$ fixed;

(G3) $G(x, \xi)=0$ for $\xi \in \partial \Omega$ and $x \in \Omega$.

Since $L$ is self-adjoint, Green's function is symmetric, in the sense that

$$
G(x, \xi)=G(\xi, x), \quad \forall x, \xi \in \Omega .
$$

As noticed in [9, pp. 87-88], a Green function with properties (G1)-(G3) exists for an operator $L$ as above if the coefficients of $L$ and the boundary of $\Omega$ are sufficiently smooth and in addition the problem

$$
\begin{cases}L u(x)=h(x), & \text { for } x \in \Omega \\ u(x)=g(x), & \text { for } x \in \partial \Omega\end{cases}
$$

has a unique solution for suitable data. Under these circumstances a solution $u$ of equation (5) is given by the formula:

$$
u(\xi)=-\int_{\Omega} G(x, \xi) h(x) d x-\int_{\partial \Omega} g(x) \frac{\partial G(x, \xi)}{\partial v_{x}} d \sigma(x) .
$$

The main result of this paper is the following theorem.

TheOREM 1. Assume $p \in C^{0, \alpha}(\bar{\Omega})$, for some $\alpha \in(0,1)$. Then a necessary and sufficient condition for the inequality

$$
\int_{\Omega} f(x) p(x) d x \leq \int_{\partial \Omega} f(\xi) \cdot\left[-\int_{\Omega} \frac{\partial G(\xi, x)}{\partial \gamma_{\xi}} p(x) d x\right] d \sigma(\xi)
$$

to hold for all $f \in C^{2, \alpha}(\bar{\Omega})$ with

$$
L f(x) \geq 0, \quad \forall x \in \Omega,
$$

is that the solution of the Dirichlet problem

$$
\begin{cases}L v(x)=p(x), & \text { for } x \in \Omega \\ v(x)=0, & \text { for } x \in \partial \Omega\end{cases}
$$

satisfies $v(x) \leq 0$ for all $x \in \Omega$. 
Here, $G(x, \xi)$ is Green's function for the operator $L$ on the domain $\Omega$, and $\frac{\partial}{\partial \gamma}$ denotes the derivative in direction $\gamma=\left(\gamma_{1}, \ldots, \gamma_{N}\right)$.

REMARK 1. Problem (9) has a unique solution via [3, Theorems 6.8 and 4.3]. Furthermore, if $p(x) \geq 0$ for all $x \in \Omega$, then the maximum principle (see [3, Corollary 3.2]) implies that solution $v$ is negative in $\Omega$.

REMARK 2. There exist functions $p(x)$ which may take negative values in $\Omega$ and such that problem (9) still has a negative solution. Indeed, in the particular case when $\Omega$ is the unit ball centered in the origin of $\mathbb{R}^{N}, L=\Delta$ (the Laplace operator), and $p(x)=|x|^{2}-\frac{N}{6}$, the solution of problem (9) is given by

$$
v(x)=\frac{|x|^{2}\left(|x|^{2}-1\right)}{12}<0, \quad \forall x \in B_{1}(0) .
$$

REMARK 3. Theorem 1 extends both the right hand side inequalities in (1) and (3). The boundary measure associated to $p(x) d x$ appears to be $\left[-\int_{\Omega} \frac{\partial G(\xi, x)}{\partial \gamma_{\xi}} p(x) d x\right] d \sigma(\xi)$.

REMARK 4. It is worth noticing that Theorem 1 can be easily extended to the general framework of signed Borel measures. For this it suffices to replace the Dirichlet problem (9) by a similar problem having the right-hand side a measure.

2. Proof of Theorem 1. Inequality (7) is equivalent to

$$
0 \geq \int_{\Omega}\left[f(x)+\int_{\partial \Omega} \frac{\partial G(\xi, x)}{\partial \gamma_{\xi}} f(\xi) d \sigma(\xi)\right] p(x) d x .
$$

Since $f \in C^{2, \alpha}(\bar{\Omega})$ it follows that $L f \in C^{0, \alpha}(\bar{\Omega})$ and thus by [3, Theorems 6.8 and 4.3] we infer that $f$ is the unique solution of the problem

$$
\begin{cases}L w(x)=L f(x), & \text { for } x \in \Omega \\ w(x)=f(x), & \text { for } x \in \partial \Omega\end{cases}
$$

Hence, by (6), we get

$$
f(x)=-\int_{\Omega} G(\xi, x) L f(\xi) d \xi-\int_{\partial \Omega} \frac{\partial G(\xi, x)}{\partial \gamma_{\xi}} f(\xi) d \sigma(\xi),
$$

and (10) is equivalent to

$$
0 \geq \int_{\Omega}\left[-\int_{\Omega} G(\xi, x) p(x) d x\right] d \xi
$$

A new appeal to formula (6) yields

$$
\begin{aligned}
v(\xi) & =-\int_{\Omega} G(x, \xi) p(x) d x \\
& =-\int_{\Omega} G(\xi, x) p(x) d x
\end{aligned}
$$

taking into account the symmetry of $G$. 
Consequently, relation (12) can be restated as

$$
0 \geq \int_{\Omega} v(\xi) L f(\xi) d \xi
$$

Or, $L f \geq 0$ in $\Omega$ and $L f$ runs over $C^{0, \alpha}(\bar{\Omega})\left(\supset C_{0}(\Omega)\right)$ when $f$ runs over $C^{2, \alpha}(\bar{\Omega})$. Thus, the last inequality holds true if and only if $v \leq 0$ over $\Omega$.

The proof of Theorem 1 is complete.

3. A particular case. In this section we point out once more the connection between Theorem 1 and the Hermite-Hadamard inequality. To do that we consider the particular case where $L=\Delta, \Omega=B_{R}(0)$ (the ball of radius $R$ centred in the origin in $\left.\mathbb{R}^{N}(N \geq 2)\right)$ and $p(x) \equiv 1$ in $\bar{\Omega}$.

We denote by $E(x)$ the fundamental solution of the Laplace equation on $\mathbb{R}^{N}$ (see $[4$, p. 8$])$, that is

$$
E(x)= \begin{cases}\frac{1}{(2-N) \omega_{N}} \cdot \frac{1}{|x|^{N-2}}, & \text { if } N \geq 3, x \neq 0 \\ \frac{1}{2 \pi} \cdot \ln (|x|), & \text { if } N=2, x \neq 0\end{cases}
$$

where $\omega_{N}$ represents the area of the unit ball in $\mathbb{R}^{N}$.

Then it is known (see [4]) that Green's function for $N \geq 3$ is given by the formula

$$
G(x, \xi)= \begin{cases}\left(\frac{R}{|x|}\right)^{N-2} \cdot E\left(x^{\star}-\xi\right)-E(x-\xi), & \text { for } x \in B_{R}(0) \backslash\{0\} \\ \frac{1}{(2-N) \omega_{N} R^{N-2}}-E(\xi), & \text { for } x=0\end{cases}
$$

while Green's function for $N=2$ is given by the formula

$$
G(x, \xi)= \begin{cases}\frac{1}{2 \pi} \cdot\left(-\ln (|x-\xi|)+\ln \left(\left|x^{\star}-\xi\right| \cdot|x| / R\right)\right), & \text { for } x \in B_{R}(0) \backslash\{0\} \\ \frac{1}{2 \pi}(-\ln (|\xi|+\ln (R))), & \text { for } x=0\end{cases}
$$

where $x^{\star}=R^{2} /|x|^{2} x$, for all $x \in B_{R}(0) \backslash\{0\}$.

A simple computation (see [4, p. 13]) shows that the normal derivative of Green's function is given by

$$
\frac{\partial G(x, \xi)}{\partial \nu_{\xi}}=\frac{|x|^{2}-R^{2}}{R \omega_{N}|x-\xi|^{N}},
$$

for all $N \geq 2$.

By Theorem 1 we infer that for any function $f \in C^{2, \alpha}(\bar{\Omega})$ with $\Delta f \geq 0$ in $\Omega$ the following inequality holds:

$$
\begin{aligned}
& \frac{1}{\operatorname{Vol} \bar{B}_{R}(0)} \int_{B_{R}(0)} f(x) d x \\
& \leq \int_{\partial B_{R}(0)} f(\xi) \cdot\left[\frac{1}{\operatorname{Vol} \bar{B}_{R}(0)} \int_{B_{R}(0)}\left(\frac{R^{2}-|x|^{2}}{\omega_{N} R}\right) \frac{1}{|x-\xi|^{N}} d x\right] d \sigma(\xi) .
\end{aligned}
$$


The above inequality is a Hermite-Hadamard type inequality since for any $x \in$ $B_{R}(0)$ we have

$$
\frac{R^{2}-|x|^{2}}{\omega_{N} R} \int_{\partial B_{R}(0)} \frac{1}{|x-\xi|^{N}} d \sigma(\xi)=1 .
$$

The last equality is an immediate consequence of Poisson's formula (see [4, p. 14-15]) and of the fact that the unique solution of the Dirichlet problem

$$
\begin{cases}\Delta u(x)=0, & \text { for } x \in B_{R}(0) \\ u(x)=1, & \text { for } x \in \partial B_{R}(0)\end{cases}
$$

is $u \equiv 1$ via the maximum principle.

More generally, relation (14) still works for a weighted Lebesgue measure, $p(x) d x$, where $p(x)$ satisfies a Dirichlet problem of the type (9). In that situation $\operatorname{Vol}\left(\bar{B}_{R}(0)\right)$ must be replaced by $\int_{B_{R}(0)} p(x) d x$.

An Open Problem. Based on the above considerations, it seems very likely that the main result of this paper remains valid for all operators $L$ that possess a Green function. In particular, for the biharmonic operator in $\mathbb{R}^{2}$ (see, e.g. [8, p. 194]),

$$
\nabla^{4}=\frac{\partial^{4}}{\partial x^{4}}+2 \frac{\partial^{4}}{\partial x^{2} \partial y^{2}}+\frac{\partial^{4}}{\partial y^{4}} .
$$

ACKNOWLEDGMENTS. The second author was partially supported by the grant CEEX 05-D11-36.

\section{REFERENCES}

1. A. M. Fink, A best possible Hadamard inequality, Math. Inequal. Appl., 1 (1998), 223-230.

2. A. Florea and C. P. Niculescu, A Hermite-Hadamard inequality for convex-concave symmetric functions, Bull. Soc. Sci. Math. Roum., 50 (98) (2007), No. 2.

3. D. Gilbarg and N. S. Trudinger, Elliptic partial differential equations of second order (Springer-Verlag, 1998).

4. J. Jost, Partial differential equations (Springer-Verlag, 2000).

5. P. Montel, Sur les fonctions convexes et les fonctions sousharmonique, Journal de Math., (9), 7 (1928), 29-60.

6. C. P. Niculescu and L.-E. Persson, Old and new on the Hermite-Hadamard inequality, Real Anal. Exchange, 29 (2003/2004), 663-686.

7. C. P. Niculescu and L.-E. Persson, Convex functions and their applications. A contemporary approach, CMS Books in Mathematics vol. 23 (Springer-Verlag, 2006).

8. J. Ockendon, S. Howison, A. Lacey and A. Movchan, Applied partial differential equations (Oxford University Press, 2003).

9. M. H. Proter and H. F. Weinberger, Maximum principles in differential equations (Springer-Verlag, 1984). 